\title{
OVERLAP OF BICKERSTAFF ENCEPHALITIS AND GUILLAIN-BARRÉ SYNDROME IN A PATIENT WITH SYSTEMIC LUPUS
}

\author{
Daniel P.A. Santos, Mariana Spitz, Perola Oliveira, Thiago Barcia, \\ Rafael Souza da Silva, Luiza Lopes de Azevedo, Claudio Meilman, \\ Luiz F. Vasconcellos, Clinete Sampaio Lacativa, Jorge Noujaim El-kadum
}

Systemic lupus erythematosus (SLE) is an auto-immune disease with multiple systems dysfunctions. Neurological complications are frequently related to the disease'. However, Guillain-Barré syndrome (GBS) and its subtypes are seldom associated to SLE 2 .

We report a rare case of association between active SLE and overlap of Bickerstaff brainstem encephalitis (BBE) and GBS, with a severe course.

\section{CASE}

A 28 year old female was admitted to the hospital due to malaise, fatigue, proximal tetraparesis, numbness, paresthesias and sluggish stretch reflexes. In addition, she presented other neurological signs including progressive ophthalmoparesis within two weeks and ataxia. A history of abdominal pain, nausea, vomits and diarrhea precede a week first neurological symptoms.

A rheumatologist consultant prescribed high dose corticosteroids, based upon signs of SLE reactivation. However, patient developed worsening of neurological symptoms. She developed drowsiness, worse weakness on limbs and neck flexion. A head $\mathrm{CT}$ scan showed no abnormalities.

Cerebro-spinal-fluid (CSF) analysis showed marked albumincytologic dissociation. Protein level was $464 \mathrm{mg} / \mathrm{dL}$ with only 5 cells. She was then admitted to Intensive Care Unit due to ventilatory failure associated to complete ophthalmoplegia and tetraplegia. Therefore, a course of plasma exchange (PE) was performed.

In addition, after PE treatment she was submitted to a 0.4 $\mathrm{g} / \mathrm{kg} /$ day Intravenous Immunoglobulin course, during 5 days. A new head CT scan and MRI were unrevealing and she underwent to coma with no brainstem reflexes. Anti-gangliosides antibodies (antiGQ1b; anti-GM1 and anti-GM2) assays were performed with negative results. She was then submitted to an electroencephalogram (EEG) that showed general slightly slowed cortical activity.

Nerve conduction studies (NCS) showed marked motor nerve abnormalities with reduced amplitude associated to $\mathrm{F}$ wave absence in four limbs. Sensory NCS showed also abnormalities characterizing a severe axonal motor and sensory polyradiculoneuropathy.

Therefore, under this severe progression, she was submitted to another IVIg course. However, she remained in coma without any brainstem reflexes, including bilateral areflexical mydriatic pupils. She died after multiple organ dysfunction due to pulmonary sepsis.

\section{DISCUSSION}

The typical form of GBS is presented in roughly $90 \%$ of the cases. Other forms that share similar pathogenesis are as follow: the Miller-Fisher syndrome, faringeal-armfacial form, acute disautonomy and the Bickerstaf brainstem encephalitis.

We describe a patient with a polyrradiculoneuropathy associated to SLE activation. This is an already rare association of diseases ${ }^{2}$. The presentation of relatively symmetrical, progressive ophthalmoplegia, ataxia, consciousness disturbance or pyramidal tract signs and limb strength with 5 or 4 on the MRC scale are features criteria for the diagnosis of $\mathrm{BBE}^{3}$. However, patients with limb weakness ( $\leq 3$ on the MRC scale), in addition to consciousness disturbance or pyramidal signs as well as ophthalmoplegia fulfill criteria for overlap of BBE and $\mathrm{GBS}^{3}$. The atypical association, in this case, with bilateral areflexical mydriasis was already reported in MFS, not in $\mathrm{BBE}^{4}$.

Hence, this patient fulfilled the criteria to characterize her presentation as overlap of BBE and GBS. This is also true once other diagnoses were rule out. Some authors have been proposing a similar classification, naming this group as "Anti-GQ1b Syndromes". It includes the GBS with ophthalmoplegia, MFS, acute ophthalmoplegia

\section{SOBREPOSIÇÃO DE ENCEFALITE DE BICKERSTAFF E SÍNDROME DE GUILLAIN-BARRÉ EM PACIENTE COM LUPUS SISTÊMICO}

Neurology Department, Hospital Servidores do Estado, Rio de Janeiro RJ, Brazil.

Received 20 March 2009. Accepted 20 July 2009.

Dr. Daniel P.A. Santos - Rua Cupertino Durão 104 / 701 - 22441-030 Rio de Janeiro RJ - Brasil. E-mail: santosdpa@yahoo.com.br 
and BBE. In this way, overlap GBS syndromes could be explained by conjugated action of antibodies ${ }^{5}$.

During the patient evaluation, anti-GQ1b serology was negative. Odaka et al. demonstrated that only $66 \%$ of BBE patients were positive for anti-GQ $1 b^{6}$. In addition, antibodies titers drop dramatically during the disease course. In addition, magnetic resonance imaging (MRI) of the brain showed no abnormalities. Odaka et al. showed only in $1 / 3$ of 62 BBE patients MRI abnormalities ${ }^{6}$.

Some infectious agents have already been involved in these cases, more commonly Campylobacter jejuni (CJ) ${ }^{6}$. However, her serology test against CJ antibodies was negative. These data confirm the low test sensitivity for the diagnosis of previous contact with $\mathrm{CJ}$ in BBE patients, approximately $22 \% 6$.

This patient showed enough criteria for the SLE diagnosis and related to lupus' nephritis, which are probably related to this severe prognosis. Finally, this patient had a positive anti-cardiolipin serology anterior to the her neurological disease. This antibody has already been described in association to GBS and hypothesized a pathogenic role ${ }^{7}$.

In conclusion, we presented a rare description of BBE in association to SLE, based upon literature researched. The association to SLE in activity brought more severe prognosis to a neurological disease with usual benign course. In addition, this association with severe prognosis demonstrates that further studies are necessary to indicate the actual association to anti-GQ1b antibody, the best definitive treatment and prognosis.

\section{REFERENCES}

1. Sedgwick RP VHK. Neurological manifestations of lupus erythematosus and periarteritis nodosa: report of 10 cases. Bull Los Angeles Neurol Soc 1948;13:129-142.

2. Robson MG WM, Davies KA. Systemic lupus erythematous and acute demyelinating polyneuropathy. Br J Rheumatol 1994;33:1074-1077.

3. Odaka M, Yuki N, Hirata K. Anti-GQ1b IgG antibody syndrome: clinical and immunological range. J Neurol Neurosurg Psychiatry 2001;70:50-55.

4. Caccavale AM, Mignemmi L. Acute onset of a bilateral areflexical mydriasis in Miller-Fisher syndrome: a rare neuro-ophthalmologic disease. J Neuroophthalmol 2000;20:61-62.

5. Willinson HJ. Ganglioside complex as targets for antibodies in Miller Fisher syndrome. J Neurol Neurosurg Psychiatry 2006;77:1002-1003.

6. Odaka M, Yuki N, Yamada M, et al. Clinical features of 62 cases and a subgroup associated with Guillain-Barré syndrome. Brain 2003;126: 2279-2290.

7. Levine SR WK. The spectrum of neurologic disease associated with antiphospholipid antibodies. Lupus anticoagulants and anticardiolipin antibodies. Arch Neurol 1987;44:876-883. 